\title{
Dynamic acousto-optical control of confined polariton condensates: From single traps to coupled lattices
}

\author{
Alexander S. Kuznetsov, Klaus Biermann, and Paulo V. Santos \\ Paul Drude Institute for Solid State Electronics Leibniz-Institut im Forschungsverbund Berlin e. V., \\ Hausvogteiplatz 5-7, 10117 Berlin, Germany
}

(Received 6 March 2019; revised manuscript received 2 May 2019; published 26 September 2019)

\begin{abstract}
Microcavity exciton-polaritons, strongly mixed light-matter states, are promising candidates for novel solidstate photonic devices such as classical and quantum simulators, qubits, and topologically protected photonic on-chip circuitry. Although different schemes for creation of confined polariton states and lattices have been proposed, a unified approach for the advanced preparation and dynamic manipulation of individual confined states, as well as for the control of interactions between remote states, is still missing. Here, we introduce a solid-state platform for dynamic polariton control based on the combination of intracavity potential traps, defined by a spatial modulation of the microcavity thickness, with dynamic control via nonlinear polariton-polariton interactions, as well as time and spatially dependent strain fields. We first demonstrate important functionalities of the platform, such as dynamic control of the energy and internal degrees of freedom, as well as the energy matching of neighboring traps. We then show the coherent modulation and symmetry control of a lattice of coupled traps by strain fields, which enables the creation of dynamic states within the band gap of the lattice. Important characteristics of the acoustic modulation are that it maintains the coherence of macroscopic polariton states and does not depend on polariton density, thus being applicable down to the single-polariton level. These functionalities of the strongly nonlinear, tunable, and spatially compact polariton platform are well described by a theoretical framework and represent important steps towards tunable polaritonic devices based on lattices of interacting polariton traps.
\end{abstract}

DOI: 10.1103/PhysRevResearch.1.023030

\section{INTRODUCTION}

Microcavity (MC) exciton-polaritons (polaritons) are bosonic quasiparticles resulting from the strong coupling between photons and quantum well excitons [1-4]. Polaritons combine the long spatial coherence of photons with strong interparticle interactions mediated by the excitonic component. Beyond fundamental interest [5-13], polaritons have been suggested for nonlinear optical devices $[14,15]$ as well as for quantum functionalities in an all-semiconductor platform [16], such as qubits [17], topological circuits [18,19], and simulators [20,21]. These applications require confinement potentials to control the energy and density of the quasineutral polariton particles.

The very low effective mass of polaritons (typically on the order of $10^{-5}-10^{-4}$ of the free electron mass, $m_{e}$ ) results in a long spatial coherence and enables polariton confinement in interacting arrays of micrometer-sized potential traps created by structuring of the MC. Different approaches have been employed to create single, micrometer-sized traps with confined polariton states, as well as lattices of interacting polariton traps with a well-defined band structure [22-24]. In

Published by the American Physical Society under the terms of the Creative Commons Attribution 4.0 International license. Further distribution of this work must maintain attribution to the author(s) and the published article's title, journal citation, and DOI. most cases, these structures are created by patterning the MC using a lithographic process to tailor the photonic or excitonic polariton components [25-32]. The structures are normally static in the sense that they are defined during the fabrication process and cannot be changed afterwards.

It is only recently that the polariton community brought its attention to polariton lattices for classical [21,33] and quantum simulators [20] and understood the stringent experimental criteria required for their realization. Several challenges presently hinder progress towards efficient structures, e.g., (i) the small polariton-polariton interaction energy (typical values are $\sim 10 \mu \mathrm{eV} \mu \mathrm{m}^{2}$ ) [27], which calls for lattice potentials with submicrometer dimensions; (ii) decoherence resulting from potential disorder; and (iii) the absence of an efficient dynamic mechanism to control energies and interactions that operates independently of the polariton density. In addition, such lattices require tools for dynamic control of the confinement potential of a single site, as well as of the on-site and intersite interactions. Dynamic potentials and lattices for polaritons can be generated by exploring nonlinear optical effects induced by spatially modulated light beams [30,31] or by the spatially dependent strain fields, produced by a surface acoustic wave (SAW) [29]. Such tunable potentials enable control of on-site lattice energies, as well as the interaction between neighboring lattice sites. One important advantage of acoustic fields over optical fields is the possibility of tuning the potential independently of the polariton density, thus enabling polariton control down to the single-particle level, which is essential for quantum applications. 
A particularly interesting approach to achieve dynamic control consists in combining SAW fields and static polariton traps and lattices. As an example, a standing SAW with a wavelength equal to the lattice periodicity can dynamically control the on-site and intersite couplings over the whole structure without a need to access individual lattice sites. The switching between the two control schemes (i.e., the on-site or intersite coupling) can be dynamically achieved via control of the phase of a standing SAW. Furthermore, recent developments in $\mathrm{MC}$ technology have pushed the polariton coherence times to values that can exceed the oscillation period of highfrequency SAWs. The latter opens a way for exploitation of time-coherent effects analogous to the side-band modulation of single quantum levels [34,35], dynamic control of coupled states, as well as realization of tunable polariton Floquet lattices [36]. SAWs provide a tool for exploitation of the rich physics associated with dynamic localization in polariton lattices [37], ac-resonant tunneling [38], as well as for the creation of artificial magnetic fields of optical lattices of cold atoms.

In this work, we show that dynamic control of polariton lattices using acoustic fields makes it possible to address several of the problems at once. First, it enables densityindependent dynamic energy control. In particular, we demonstrate that static polariton potentials can be combined with dynamic modulation by optical and SAW fields to provide the previously unattainable dynamic control of the energy, as well as the interaction between confined polariton states. We show that modulation by SAW periodically shifts the discrete polariton levels by as much as $1.5 \mathrm{meV}$. The latter is attributed to modulation of the energies of photonic and excitonic transitions by the SAW strain field. Second, the acoustic modulation does not introduce decoherence and can extend over arbitrary large (up to millimeter) length scales. We demonstrate that the ratio between the acoustic wavelength $\left(\lambda_{\text {SAW }}\right)$ and trap size plays an important role in the character of the modulation. While acoustic fields modulate small traps as a whole, these fields can fragment large traps, leading to the formation of arbitrarily small (down to $\lambda_{\mathrm{SAW}} / 2$ ) disorder-free minitraps. Third, we demonstrate that the spatial dependence of the SAW field provides a powerful tool to dynamically tune the energy of closely spaced traps. In particular, traps separated by $\lambda_{\mathrm{SAW}} / 2$ can be periodically brought in and out of resonance by acoustic modulation.

Finally, we present experimental evidence for dynamic control of the dispersion of a lattice of coupled static traps. For that purpose, we use a SAW with an amplitude comparable to the Rabi energy to create new dynamic polariton states localized in the energy gap of the static lattice. These results also prove that acoustic modulation preserves the long spatial coherence of polariton condensates. The experimental findings are well accounted for by a theoretical framework, thus proving a high degree of controllability of this dynamic polariton system.

\section{RESULTS}

In this section, we first address the basic functionalities of the acoustically controlled polariton platform (Secs. II A and II B) and then combine those to demonstrate the dynamic control of polariton lattices (Sec. II C).

\section{A. Light-induced energy control}

The micrometer-sized intracavity traps for polaritons studied here were defined by structuring an $(\mathrm{Al}, \mathrm{Ga}) \mathrm{As} \mathrm{MC}$ in between subsequent molecular beam epitaxial growth runs [25-32]. The sample was designed to be in the strongcoupling regime both in the trap region (i.e., regions with thicker spacer) as well as in the surrounding barrier regions with thinned spacer. The latter enables optical excitation of high polariton densities in the intracavity trap and surrounding barriers. We have demonstrated in Ref. [32] that the intracavity traps support polariton condensates at high optical excitation density. Details of the sample structure as well as of the spectroscopic characterization methods are described in Appendixes A 1 and A 2, respectively.

In the following we show that the special design of the sample enables optical control of both trap and barrier energies via nonlinear polariton interactions. The lateral confinement induces the formation of discrete energy states within the traps. Figure 1(a) displays a photoluminescence (PL) map of a $4 \times 4 \mu \mathrm{m}^{2}$ trap recorded under low polariton excitation density (i.e., 3 orders of magnitude below the polariton condensation threshold, $P_{t h}=13 \pm 2 \mathrm{~kW} / \mathrm{cm}^{2}$ for the excitation energy $\left.E_{\mathrm{Exc}}=1.596 \mathrm{eV}\right)$. The first three confined levels can be clearly identified. The emission from the higher levels merge with that of the barrier around the trap with energy $E_{\text {Barrier }}=1534 \pm 0.5 \mathrm{meV}$ (denoted by the red arrow).

Figure 1(b) shows a time-integrated PL spectrum of the same trap excited at $3 \times P_{t h}$. The high excitation density was chosen to create condensates not only in the ground state but also in the higher confined levels. The measured PL linewidth of the lowest level reduces to $\Delta E_{1}=0.116 \pm$ $0.002 \mathrm{meV}$ upon the condensation, thus becoming approximately 3 times smaller than that for the noncondensed polaritons. This condensate linewidth is most likely limited by the spectral resolution of our detector $(0.085 \mathrm{meV})$. However, dynamic potential fluctuations around the trap may introduce additional spectral diffusion. Together with the linewidth narrowing and the enhancement of the PL intensity by several orders of magnitude, the condensate energy levels are blueshifted by $\Delta E_{\text {Cond.-Blueshift }}=1.72+/-0.05 \mathrm{meV}$ and also have an increased interlevel separation with respect to the ones below the condensation threshold. The latter is indicative of an increase of the confinement energy under a high polariton density due to a pronounced blueshift of the barrier states. Indeed, the red arrows in Figs. 1(a) and 1(b) show that the barrier energy blueshifts upon the condensation by $\Delta E_{\text {Barrier }} \approx 6 \pm 0.6 \mathrm{meV}$ (i.e., from $E_{\text {Barrier }}=1534 \pm$ $0.5 \mathrm{meV}$ to $E_{\text {Barrier_Cond }} \approx 1540 \pm 0.1 \mathrm{meV}$, the error represents half-width at half maximum of the respective levels), resulting in larger energy separation of the confined levels. These effects show that the polariton character of the barriers enables control of the trap energy profiles via the excitation density.

\section{B. Modulation of single-polariton traps by SAWs}

For dynamic acoustic control, the polariton traps were placed within an acoustic resonator defined by a delay line with two interdigitated transducers (IDTs), as illustrated in 


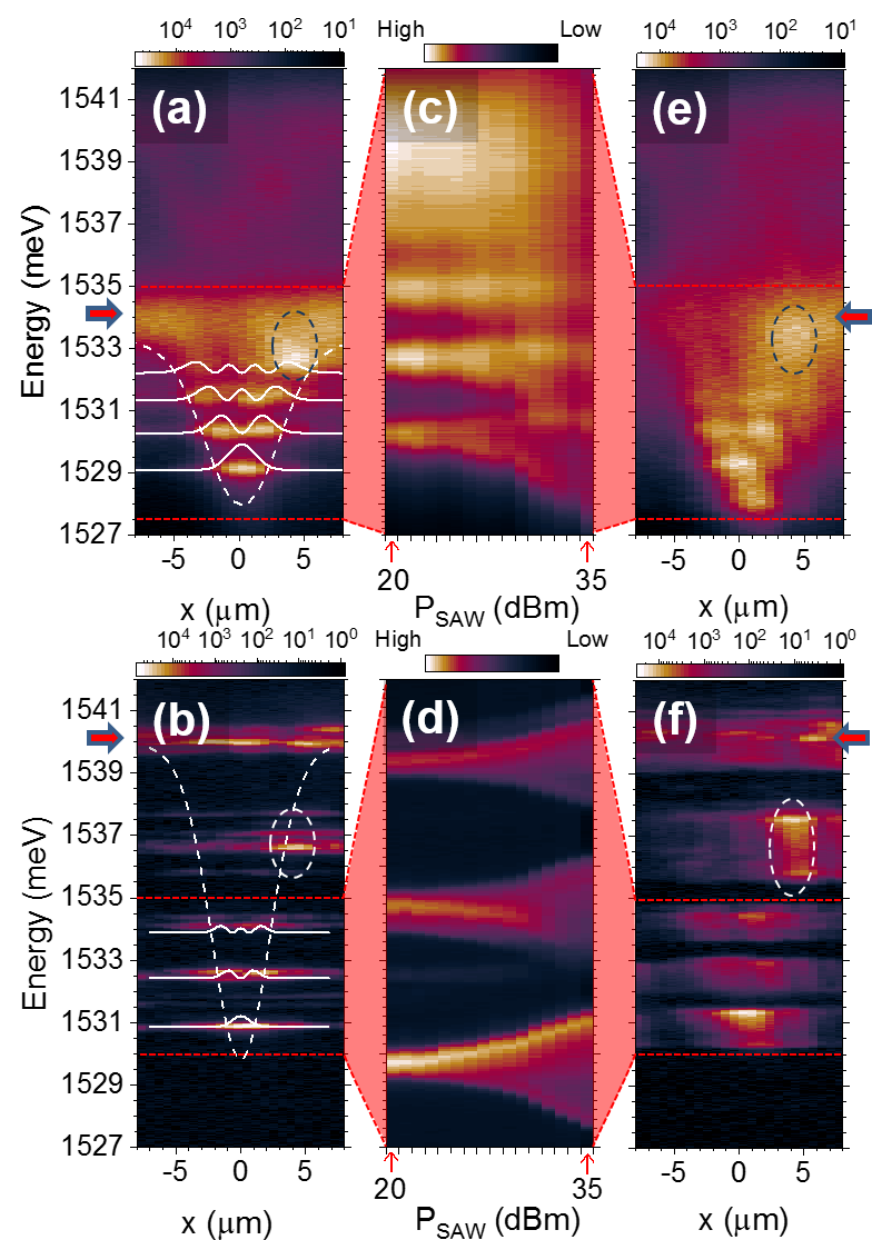

FIG. 1. (a), (b) Spectral PL maps of a $4 \times 4 \mu \mathrm{m}^{2}$ trap optically excited at (a) $0.001 \times P_{t h}$ and (b) $3 \times P_{t h}$. (c), (d) The corresponding evolution of the spatially integrated emission of the trap as the SAW power $P_{\text {SAW }}$ increases from $20 \mathrm{dBm}\left(1.6 \times 10^{-4} \mathrm{~W} / \mathrm{m}\right)$ to $35 \mathrm{dBm}$ $(49.4 \mathrm{~W} / \mathrm{m})$ in steps of $1 \mathrm{dBm}$. The energy scale in (c) is from 1528 to $1535.5 \mathrm{meV}$ and in (d) is from 1530 to $1535 \mathrm{meV}$. (e), (f) PL maps similar to (a) and (b) but recorded at $P_{\mathrm{SAW}}=35 \mathrm{dBm}$. The lowest energy levels are confined states within the trap. The horizontal red arrows in (a), (e), (b), and (f) indicate the energy of the barrier, which is blueshifted at high excitation powers. The emission marked by the dashed ellipsis arises from a 0.6- $\mu$ m-wide strip located at $x \approx$ $5 \mu \mathrm{m}$. The dashed and solid lines in (a) and (b) represent calculated potential shape and wave functions of the trap, respectively.

Fig. 2(a) and described in detail in Appendix B 1; the resonator configuration enables the confinement of the acoustic field, thus enhancing the interaction with polaritons. The SAWs were excited along the $x=[-110]$ direction of the $z=$ (001) MC surface using IDTs fabricated by the optical lithography. The single-finger IDTs were designed for the acoustic wavelength $\lambda_{\text {SAW }}=8 \mu \mathrm{m}$ (corresponding to the frequency $f_{\text {SAW }}=383 \mathrm{MHz}$ at $10 \mathrm{~K}$ ). As displayed schematically in Fig. 2(b), the acoustic modulation of the intracavity traps is due to the modulation of the spacer and quantum well (QW) thicknesses by the evanescent strain of the SAW. The value of $\lambda_{\text {SAW }}$ was selected to maximize the strain-induced band-gap modulation at the depth of the QWs.
The modulation of the photonic and excitonic levels was calculated from the strain profiles obtained from an elastic model for the MC structures (cf. Appendix B 2). The calculations predict that the electron-heavy hole (e-hh) transition energy oscillates with an amplitude of approximately $\pm 0.8 \mathrm{meV}$ around the unperturbed band-gap energy. The same calculations show that the expected SAW modulation of the optical MC resonance energy $\left(\Delta E_{c}\right)$ is approximately 3 times smaller than that of e-hh transition. The SAWs propagate along the piezoelectric [-110]-direction of the (001) surface of the MC and thus carry a piezoelectric field. This field can dissociate excitons, inducing polariton decoherence. We will show, however, that this field becomes screened at large polariton densities.

The SAW was excited by driving one of the IDTs with $f_{\mathrm{SAW}}=383.69 \mathrm{MHz}$, corresponding to one of the narrow resonances in the acoustoelectric response (S11), see Fig. 2(c). The standing character of the SAW is evidenced by the acoustic echoes in the time-domain trace of S11, cf. Fig. 2(d). The maximum applied acoustic power was $P_{1}=50 \mathrm{~W} / \mathrm{m}$, as detailed in Appendix B 1.

The standing SAW field localized between the IDTs induces a spatial modulation of the PL intensity, which can be probed by time- and energy-integrated PL. Figures 2(e) and 2(f) show PL maps of a MC region between the IDTs, recorded in the absence and in the presence of a SAW, respectively. The maps were obtained by spectrally integrating the PL intensity over the polariton emission range. The map recorded with a SAW displays a series of bright stripes with a periodicity along the $x$ direction equal to $\lambda_{\text {SAW }} / 2=4 \mu \mathrm{m}$. Therefore, the position of traps relative to the standing field can be inferred directly from PL maps, as illustrated for the two $4 \times 4 \mu \mathrm{m}^{2}$ intracavity traps marked by dashed circles, cf. Figs. 2(e) and 2(f). A comparable spatial PL modulation has been reported for QWs subjected to a standing SAW field and attributed to the enhanced exciton dissociation at the antinodes of the longitudinal piezoelectric field created by the SAW [39-42]. A similar mechanism applies to the present case, as discussed in detail in Appendix C. In fact, a comparison between the PL intensities in Figs. 2(e) and 2(f) shows that the overall PL intensity from the polariton states is significantly reduced under the excitation by SAW, in agreement with studies reported in Ref. [43].

\section{Dynamic modulation of confined polaritons}

In the previous section, we addressed only the spatial modulation of the PL intensity by the standing SAW field. The SAW modulation also manifests itself in time-integrated PL spectra as an apparent splitting of the discrete polariton levels by as much as $1.5 \mathrm{meV}$. Figures 1(c) and 1(d) compare the evolution of the time-integrated PL spectrum of the same $4 \times 4 \mu \mathrm{m}^{2}$ trap with increasing SAW powers $\left(P_{\mathrm{SAW}}\right)$ in the noncondensed and condensed regimes, respectively. These spectra were obtained by integrating the PL intensity over the lateral extension of the trap. The acoustic modulation induces an apparent energy splitting of each confined level into a doublet, which becomes more pronounced at the higher SAW powers. The energy splitting is asymmetric with respect to the energy of the unperturbed state in the absence of a SAW. 

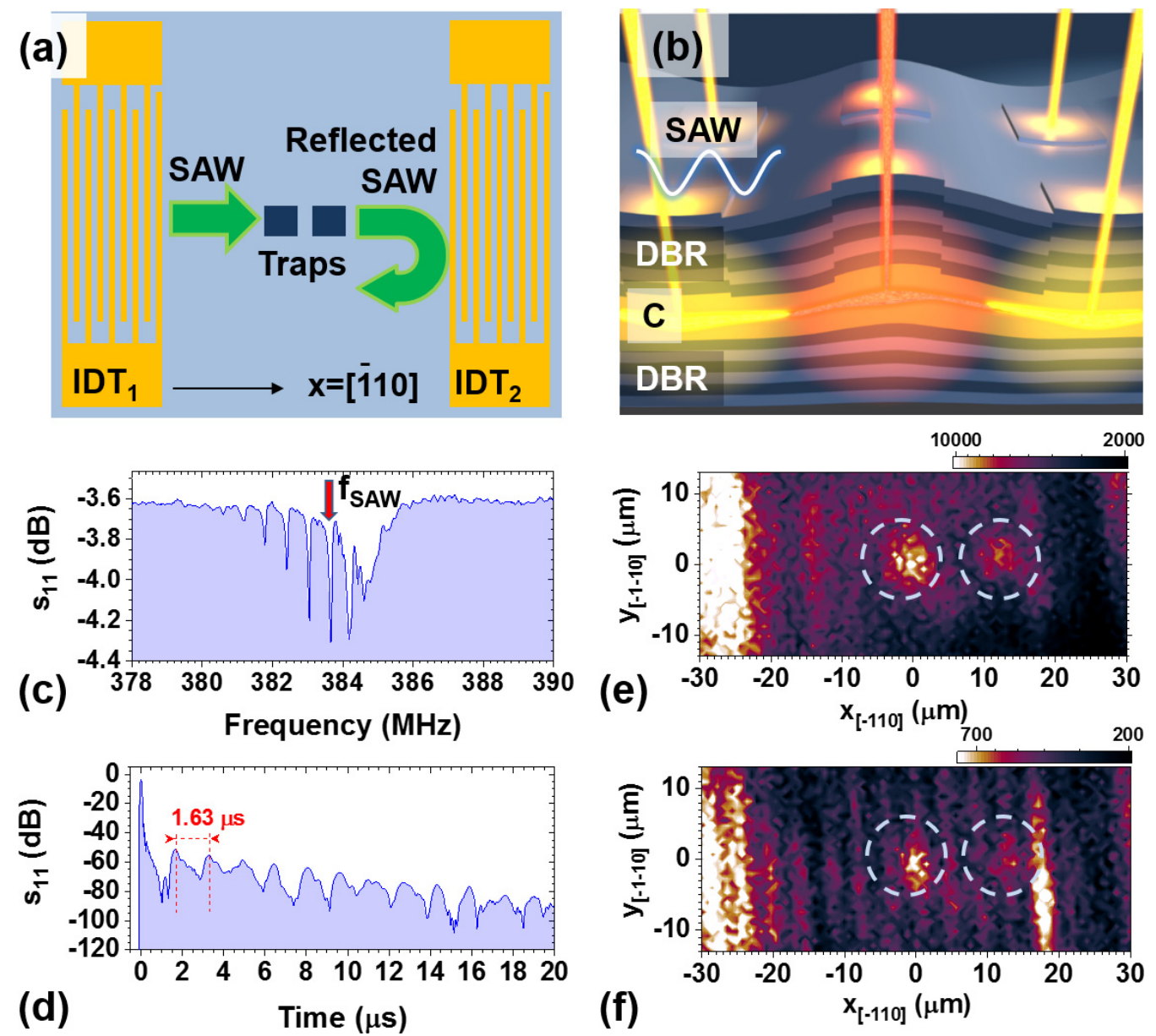

FIG. 2. (a) A sketch of a $(\mathrm{Al}, \mathrm{Ga}) \mathrm{As} \mathrm{MC}$ with intracavity traps modulated by a SAW. The traps are formed by the lateral thickness modulation of the MC spacer (C) between the DBRs. (b) A top-view sketch of the MC showing the SAW delay line resonator defined by two IDTs. The SAW with a wavelength of $\lambda_{\mathrm{SAW}}=8 \mu \mathrm{m}$ is generated along the $x \|[-110]$ direction by IDT $_{1}$ (green arrow) and backreflected by $\mathrm{IDT}_{2}$. (c) Frequency and (d) time dependence of the $s_{11}$ rf parameter for IDT 1 at $10 \mathrm{~K}$. The multiple echoes in (d) are delayed by the round-trip time $t_{r}=1.63 \mu \mathrm{s}$ for the acoustic cavity formed by the IDTs. (e), (f) Energy- and time-integrated PL maps of the area around the trap in (b) recorded in the (e) absence and (f) presence of a SAW. The PL was excited by a wide laser spot with intensity below the condensation threshold. The trap positions are indicated by the dashed circles. The vertical lines displaced by $\lambda_{\mathrm{SAW}} / 2=4 \mu \mathrm{m}$ result from modulation of the PL by the standing SAW within the acoustic resonator.

This asymmetry becomes more pronounced for high acoustic intensities and low optical excitation levels [see Fig. 1(c)], as the lowest doublet (the ground-state) components strongly redshift with increasing acoustic powers. Furthermore, the higher-energy doublet component has a higher emission intensity than the lower one. Finally, Figs. 1(e) and 1(f) also show that the acoustic field modulates not only the confined states but also the energy levels of the barrier (indicated by the red arrows). In our system, therefore, the barrier energy can thus be controlled via both optical excitation and acoustic modulation.

To understand the mechanisms responsible for the acoustic modulation, we have measured the time dependence of the PL emission from the confined condensate levels of the $4 \times 4 \mu \mathrm{m}^{2}$ trap of Fig. 1(b). Figure 3(a) shows PL spectra without (the black curve) and under the SAW excitation (the red curve), obtained by integrating over the spatial coordinate (from $x=-5 \mu \mathrm{m}$ to $x=5 \mu \mathrm{m}$ ) the emission of the confined condensates in Figs. 1(b) and 1(d). The time-resolved PL trace in Fig. 3(b), recorded using a streak camera, displays the corresponding temporal evolution of the trap emission under a SAW. The latter clearly shows the sinusoidal energy modulation of the confined levels by the SAW. In particular, the time integration of the time-resolved traces yields the PL spectrum with doublets illustrated by the thick red line in Fig. 3(a).

For the small trap discussed above, all the confined levels are modulated at the SAW frequency, which indicates that the SAW field affects the trap as a whole. This behavior results from the small dimensions of the trap (comparable to $\lambda_{\mathrm{SAW}} / 2$ ) and the fact that the trap is centered at an antinode of the SAW field. The peak-to-peak energy modulation amplitude in Fig. 3(b) is approximately equal to $1.25 \mathrm{meV}$ for the first and second confined levels and is reduced for the higher confined states. This behavior is attributed to the paraboliclike shape of the confinement potential [32], which increases the spatial extent of the polariton wave functions of the higher energy levels beyond $\lambda_{\text {SAW }} / 2$, thus averaging the impact of the SAW. 
(a)

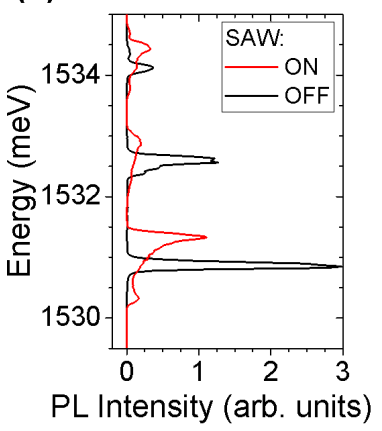

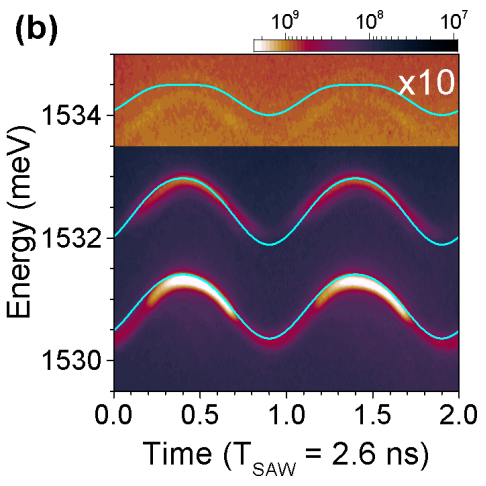

FIG. 3. (a) PL spectra of the $4 \times 4 \mu \mathrm{m}^{2}$ trap obtained by integrating the maps in Figs. 1(b) and 1(f) along the $x$ axis (from -5 to $+5 \mu \mathrm{m}$ ). (b) Time-resolved PL trace of the emission corresponding to the red-line spectrum in (a). The superimposed light-blue lines in (b) are calculated time-resolved profiles.

In order to obtain a quantitative description of the modulation amplitude, we used the spatial distribution of the SAW strain fields determined by the elastic model of Appendix B 2 to calculate the energy shifts of the bare exciton and photon cavity modes. The excitonic energies were determined by taking into account the static profile of the traps [32], as well as the deformation-potential energy shifts induced by the SAW strain field. The static potential profiles of the traps were determined from atomic force micrographs (AFMs) of the sample surface [32]. The photonic shifts were calculated by taking into account the modulation of the thickness and refractive index of the MC by the strain field. The polariton energy levels and wave functions were then determined by introducing the photon-exciton coupling using the measured Rabi splitting. Details of the calculation procedure are described in Appendix D.

At low particle density (low optical pump), the model predicts the energy spectrum as well as the spatial extent of the polariton wave functions of the trap very accurately, as shown by the white lines in Fig. 1(a). Here, the dashed and solid white lines represent the calculated trap potential and the amplitudes of the wave functions of the confined levels, respectively. Note that the paraboliclike shape of the trapping potential results in more spatially extended wave functions for the excited states. In the condensed regime, the re-normalization of the potential energy, due to the effects discussed in Sec. II A, was taken into account in order to reproduce the spectrum in Fig. 1(b). The same model was used to calculate the time evolution of the first three confined levels in the $4 \times 4 \mu \mathrm{m}^{2}$ trap of Fig. 3(b). The results are given by the solid cyan lines superimposed on the experimental traces; they reproduce very well the main features of the time evolution of the condensates energies.

Finally, the periodic energy tuning in Fig. 3 is accompanied by a modulation of the PL intensity at the SAW frequency. In particular, the PL intensity increases by almost an order of magnitude when the level energies are blueshifted by the SAW. As discussed in detail in Appendix E, the modulation of the PL intensity is most likely due to the redistribution of carriers within the trap induced by the piezoelectric potentials of the SAW.

\section{Dynamic symmetry control}

The above results are relevant for small traps (with dimensions below $\left.\lambda_{\mathrm{SAW}} / 2\right)$, which are modulated as a whole by the acoustic field. We now turn our attention to the interaction of standing SAWs with larger traps, where the acoustic field has a significant impact on the symmetry of the confinement potential.

Figure 4(a) displays a PL map recorded across a $10-\mu \mathrm{m}$ wide and $200-\mu \mathrm{m}$-long wirelike trap under an optical excitation $10^{3}$ times below the polariton condensation threshold $\left(P_{t h}=17 \pm 3 \mathrm{~kW} / \mathrm{cm}^{2}\right)$. In this regime, the confined energy levels can be clearly identified, despite the fact that their energy separation is comparable to the spectral linewidths. The superimposed dashed and solid lines are the trap potential and wave functions, respectively, calculated according to the procedure described in Appendix D. Excitation at $5 \times P_{t h}$ results in the formation of condensates in the first five levels of the trap, as illustrated in Fig. 4(b). As we discussed in Sec. II A, the energies of these states are also modified by the light-induced shifts in the barrier and in the trap.

Spectroscopic studies under a SAW were carried out on a wire with a node of the acoustic field approximately at its center. Figure 4(c) shows the evolution of the confined levels with increasing $P_{\text {SAW }}$. Similar to the small trap [cf. Fig. 1(d)], each confined level splits into a doublet. Figure 4(d) shows a spatial PL map of the wire at the largest applied SAW power. Comparing Figs. 4(c) and 4(d) for the large trap with the corresponding figures for the small trap, Figs. 1(d) and 1(f), respectively, we note the following remarkable differences in the behavior of condensed polaritons under a SAW field: (i) the energy splitting of the doublet components becomes highly asymmetric for the large trap; (ii) the time-integrated wave function of the ground level of the large trap exhibits two lobes [indicated by red arrows in Fig. 4(d)], in contrast to the single lobe observed for the small trap [cf. Fig. 1(f)]; (iii) for the large trap, the evolution of the second confined level [at $1530.2 \mathrm{meV}$ in Fig. 4(c)] with increasing acoustic power shows an avoided crossing with the first and third levels.

The time-resolved PL traces of the wire trap, cf. Fig. 4(e), are also very different from those of the small traps of Fig. 3(b). In particular, the ground-state energy oscillates in time at twice the SAW frequency. Furthermore, the time evolution of the lowest confined levels displays a series of level anticrossings at well-defined SAW phases. Finally, the energy oscillation in time of the ground state is accompanied by a periodic shift in position of the main lobe of its wave function (more precisely, of the modulus squared $\left|\psi_{0}\right|^{2}$ ), as shown in the PL map of Fig. 4(f). The latter map shows that the two maxima indicated by arrows in the time-integrated plots of Fig. 4(d) result from the periodic shift of the wavefunction lobes between positive and negative $x$ coordinates.

The unusual time dependence of the modulation can be accurately reproduced using the numerical model for the acoustic modulation of Appendix D. Figures 4(g)-4(i) show snapshots of the spatial profiles of the potential energy and wave-function amplitudes $\left(\left|\psi_{i}\right|^{2}\right.$ for the $i$ th confined state) of the wire calculated for the three phases of the SAW corresponding to the horizontal dashed white lines in Fig. 4(f). The standing SAW with a node of the strain field at the center 

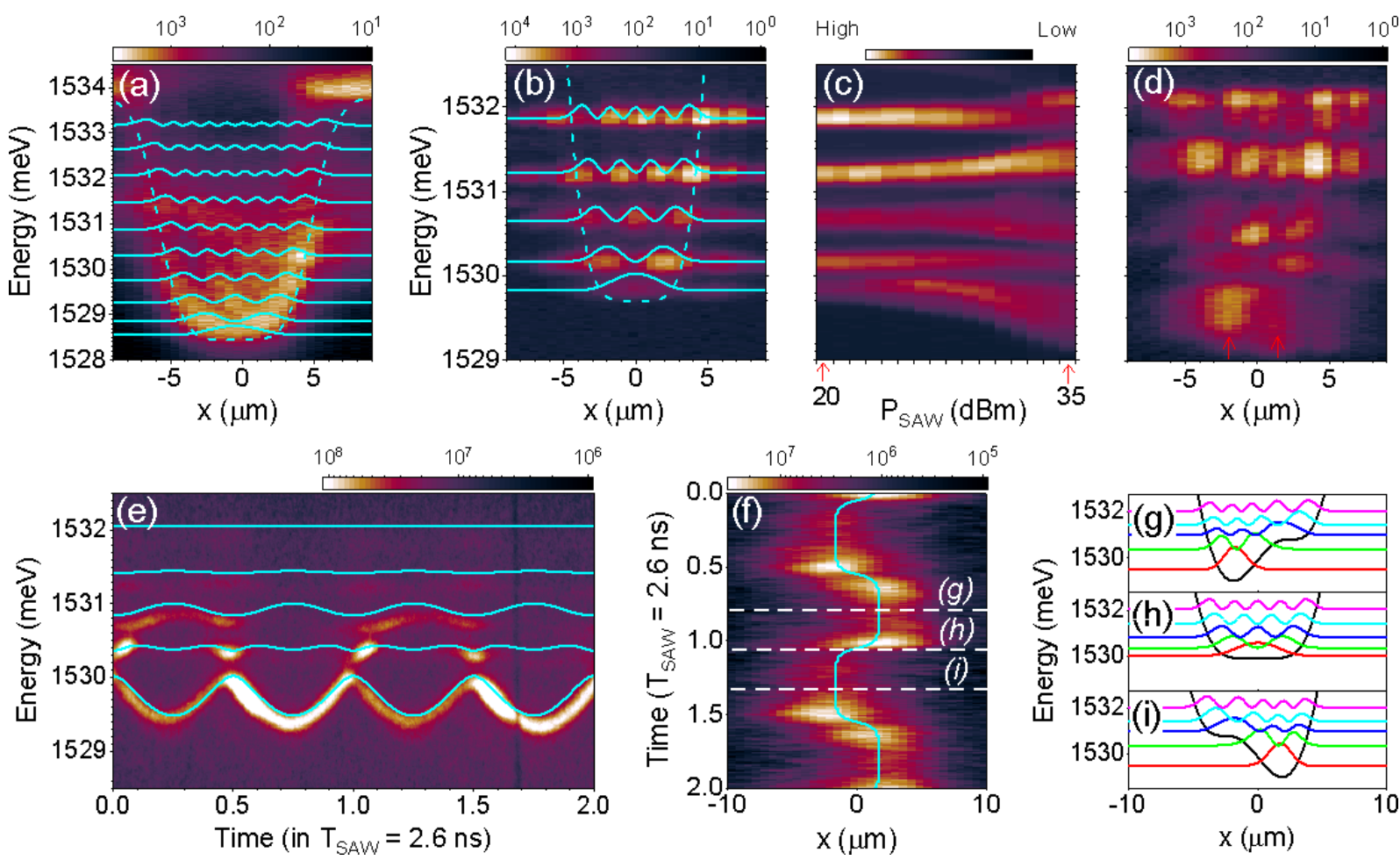

FIG. 4. (a), (b) Spectral PL maps of a 10- $\mu$ m-wide wire trap optically excited at (a) $0.001 \times P_{t h}$ and (b) $5 \times P_{t h}$ in the absence of SAW. The dashed and solid light-blue lines are calculated trap potential and wave-function amplitudes, respectively. (c) Evolution of the spatially integrated emission of the wire as the SAW power $P_{\mathrm{SAW}}$ increases from $20 \mathrm{dBm}\left(1.6 \times 10^{-4} \mathrm{~W} / \mathrm{m}\right)$ to $35 \mathrm{dBm}(49.4 \mathrm{~W} / \mathrm{m})$ in steps of $1 \mathrm{dBm}$. (d) PL map of the wire at the highest $P_{\mathrm{SAW}}$ in (c). (e) Time-resolved PL trace of the emission corresponding to the map in (d); the solid cyan lines are calculations. (f) Time evolution of the spatial position of the PL from the ground state [energy range 1529-1530 meV in panel (d)]. (g)-(i) Calculated spatial profiles of the potential and wave-function amplitudes of the trap for three different phases of the SAW standing field indicated by the horizontal dashed lines in (f). The solid cyan line in (f) displays the calculated position of the wave-function maxima for the ground state. The maps (d)-(f) were recorded under $2.5 \times P_{t h}$ and $P_{\mathrm{SAW}}=35 \mathrm{dBm}$.

of the trap periodically shifts the position of the potential minimum between positive and negative $x$ values. The latter has several important consequences. First, the lobe of $\left|\psi_{0}\right|^{2}$ periodically oscillates between positive and negative $x$ values, in agreement with the results shown in Fig. 4(f). The solid cyan line in this figure represents the calculated time evolution of the peak amplitude of the ground-level wave function extracted from plots akin to those in Figs. 4(g)-4(i). The latter accurately reproduces the time dependence of the lobe position. The second consequence is that the energy of the confined states oscillates at twice the SAW frequency. In fact, the calculated time dependence given by the solid cyan lines in Fig. 4(e) reproduce very accurately the measured energy shifts. Finally, the model also shows that the changes in the potential symmetry mix the confined levels, thus leading to the anticrossings at particular SAW phases.

\section{Dynamic energy tuning of neighboring traps}

The previous sections have addressed the effects of the SAW field on an isolated intracavity trap. In this section, we show that the spatiotemporal behavior of the SAW can also be applied to dynamically tune the energy of neighboring traps, thus providing a powerful tool to tune on-site energies as well as the intersite coupling in polariton arrays of intracavity traps. For that purpose, we will consider the acoustic modulation of a two-dimensional array of $1.6 \times 1.6 \mu \mathrm{m}^{2}$ traps with a lattice constant equal to $1.2 \times \lambda_{\text {SAW }} / 2$. Figure $5(\mathrm{a})$ shows a PL map of a single row of the array excited in the linear regime (i.e., below the condensation threshold). For low excitation densities, the ground polariton state $\left(E_{1}=\right.$ $1532.35 \pm 0.01 \mathrm{meV})$ is strongly localized within the individual traps. The first excited state of the array $\left(E_{2} \approx 1533.33 \pm\right.$ $0.01 \mathrm{meV}$ ), in contrast, is formed by the hybridization of the corresponding levels of isolated traps, creating a state with antibonding character, with the maxima of the wave-function amplitude localized between the traps [32].

Figure 5(b) shows that optical excitation of approximately $1.2 \times P_{t h} \quad\left(P_{t h}=25.5 \pm 2 \mathrm{~kW} / \mathrm{cm}^{2} \quad\right.$ for $\left.\quad E_{\mathrm{Exc}}=1.631 \mathrm{eV}\right)$ blueshifts the energy and induces condensation in the ground energy level. We note that the barrier energy also blueshifts due to the mechanisms discussed in Sec. II A. The confined condensates within the array have slightly different energies due to the unequal polariton densities arising from the finite size of the Gaussian laser spot (with a FWHM of about $18 \mu \mathrm{m})$. A SAW induces an apparent splitting of the timeintegrated PL lines of the traps by as much as $1.5 \mathrm{meV}$ [cf. Fig. 5(c)]. This splitting is not uniform and varies across the array; this is attributed to the differences in the amplitude of the standing SAW field at the location of each trap site caused by the fact that the lattice period is not exactly a multiple of $\lambda_{\text {SAW }} / 2$. 

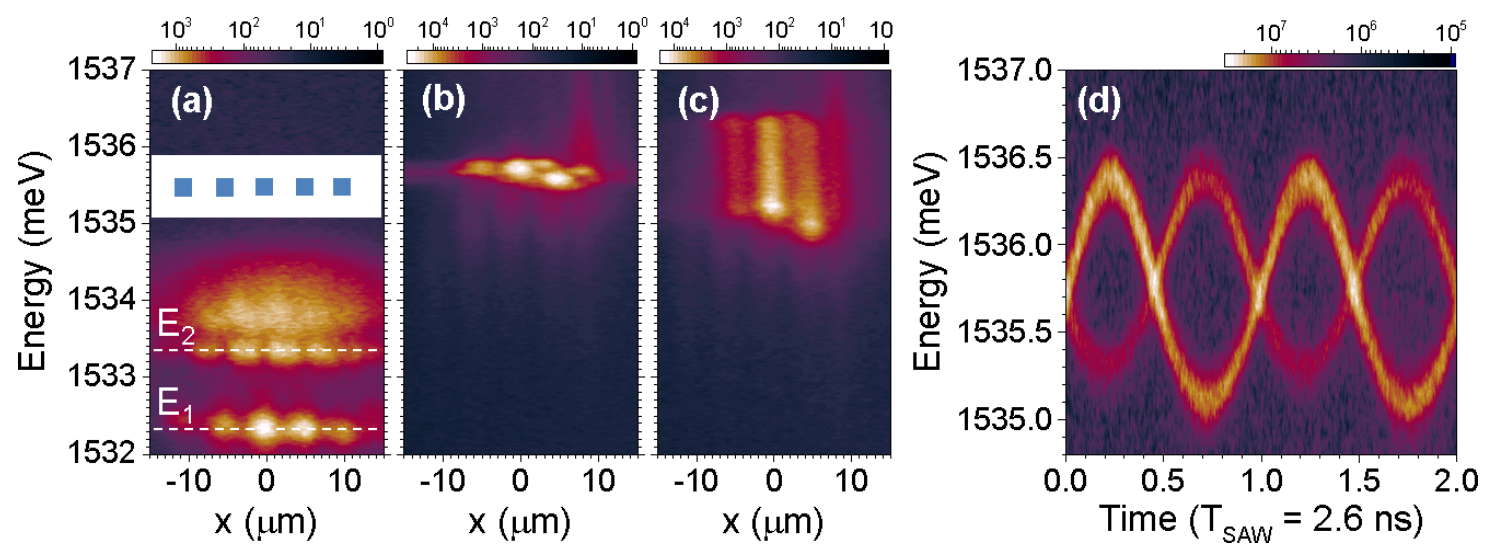

FIG. 5. A time-integrated PL map of a $4 \times 5$ array of $1.6 \times 1.6 \mu \mathrm{m}^{2}$ traps with lattice constant of $1.2 \times \lambda_{\text {SAW }} / 2$ recorded for (a) $P_{L}=$ $0.001 \times P_{t h}$ and $P_{\mathrm{SAW}}=0 \mathrm{~W} / \mathrm{m}$, (b) $P_{L}=1.2 \times P_{t h}$ and $P_{\mathrm{SAW}}=0 \mathrm{~W} / \mathrm{m}$, and (c) $P_{L}=1.2 \times P_{t h}$ and $P_{\mathrm{SAW}}=49.4 \mathrm{~W} / \mathrm{m}$. The filled squares in the inset in (a) represent the spatial locations of the traps. (d) Time-resolved PL traces measured at a position between two neighboring traps under the conditions corresponding to (c).

The dynamic energy tuning is demonstrated by the time trace of the evanescent PL, see Fig. 5(d), which was collected at a position halfway between two neighboring traps (located at $x=0$ and $x=5 \mu \mathrm{m}$ ). The emission energies of the traps undergo a mutually antiphase sinusoidal modulation. The observation of the two time traces is possible due to the overlap of the confined wave functions in the region between the traps, thus proving that the traps interact with each other. The absence of an avoided crossing at the resonance ( $t=T_{\mathrm{SAW}} / 2, T_{\mathrm{SAW}}, 3 / 2 T_{\mathrm{SAW}}, \ldots$ ) indicates, however, that the interaction energy is small compared with the linewidth of the levels, in agreement with the results of Ref. [32].

\section{Acoustic modulation of dispersion of an array of coupled traps}

In this section, we combine the functionalities of the polariton platform described in the previous sections to provide a proof of concept of the dynamical modulation of a one-dimensional (1D) lattice of polariton wirelike traps. The acoustic modulation of the lattice provides a powerful tool to study artificial polariton band structures, polariton tunneling, as well as the evolution of 1D Hamiltonians. We will show that our dynamic modulation approach offers possibilities for manipulation of the lattice dispersion far beyond the limits achievable in the static regime.

The investigation was carried out using an array consisting of $w=4.5-\mu \mathrm{m}$-wide and 200- $\mu \mathrm{m}$-long wirelike traps with a repetition period $d=5 \mu \mathrm{m}$, cf. Fig. 6(a). The sub- $\mu$ m-thick barriers separating the wires enable the coupling between polariton condensates excited in the wires. The coupling manifests itself in the time-integrated PL dispersion shown in Fig. 6(b) as the formation of energy bands separated by energy band gaps. The band gaps appear at the edges of the miniBrillouin zones at wave vectors equal to integer multiples of $k_{x \text {-array }} / 2=\pi / d$, where $k_{x \text {-array }}$ is the lattice reciprocal wave vector and $x$ is the coordinate across the array. Figure $6(\mathrm{c})$ shows that the condensate wave functions overlap in space even for the ground-state energy.

Figure 6(e) displays the time-integrated dispersion of the same lattice when subjected to a standing SAW with $\lambda_{\text {SAW }}=$ $8 \mu \mathrm{m}$, which is larger than the static modulation period (and, thus, with the acoustic wave vector $k_{x \text {-SAW }}=2 \pi / \lambda_{\text {SAW }}<$ $\left.k_{x \text {-array }}\right)$. We stress that the magnitude of the acoustically induced energy changes is comparable to static ones, thus indicating that the impact of the SAW goes beyond that of a simple perturbation. In an unstructured polariton region, this acoustic modulation folds the dispersion within miniBrillouin zones with dimension equal to $k_{x \text {-SAW }}$ [44]. In the present case, in contrast, the interplay between the static and dynamic modulation gives rise to new polariton states within the band gap of the static lattice. The localized character of these states can be inferred from the real space map of Fig. 6(f). Their energies are controlled by the SAW amplitude, while their location depends on the phase of the standing SAW field. While fixed in the current configuration, cf. Fig. 2(a), this phase, in principle, can be dynamically tuned by powering the second IDT of the delay line and controlling its rf phase relative to the first IDT. The experimental results displayed in Fig. 6(f) thus unambiguously show that acoustic fields dynamically control the energy, coupling, and symmetry of polariton condensates in lattices without affecting polariton macroscopic coherence. Implications of these results will be discussed in the next section.

The theoretical framework of Appendix D was extended to model the acoustic modulation of the 1D lattice of Fig. 6(a). The calculations were carried out using a supercell containing ten static periods and ten periods of the standing SAW field. As illustrated in Fig. 6(d), the model accurately predicts the overall energy spectrum as well as spatial character of the condensate wave functions in the absence of a SAW. In particular, the model reproduces the formation of the bands separated by the energy gaps, as well as the coupling of the excited states of the lattice. When a standing SAW having a 1.5-meV amplitude is superimposed on the static array, the model well reproduces the amplitude, as well as the spatial profile of the lattice ground state, cf. Fig. 6(g). Importantly, the model predicts localized states within the first stop band of the static lattice. According to the numerical results, the states appear only for certain spatial phases of the standing SAW with respect to the spatial profile of the array potential. Thus, the new localized states are coherent states of the lattice modified by the SAW. 
(a)

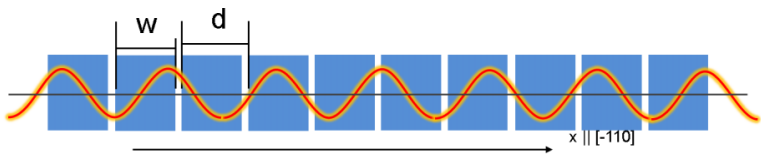

(b)
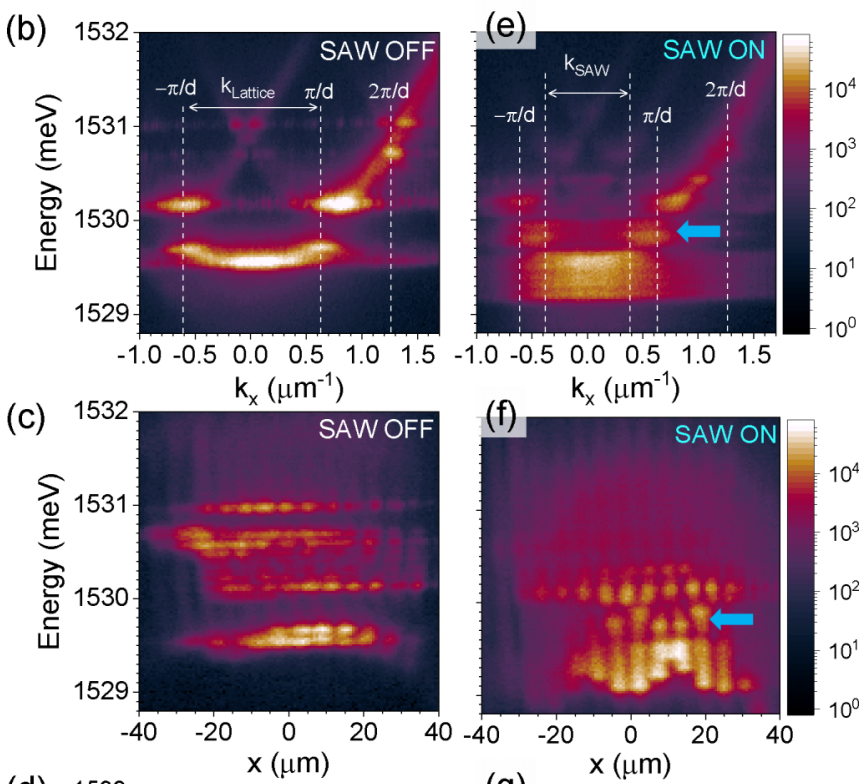

(d)

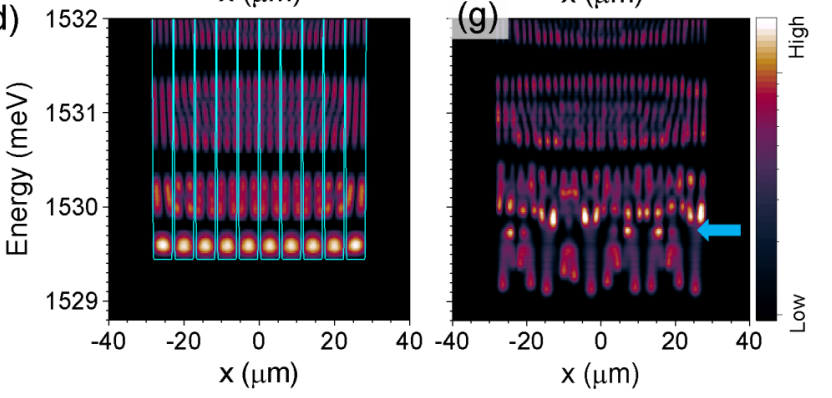

FIG. 6. (a) Schematic diagram of a one-dimensional lattice of $w=4.5-\mu \mathrm{m}$-wide and 200- $\mu \mathrm{m}$-long wire traps with periodicity $d=5 \mu \mathrm{m}$. The sinusoidal wavy red line represents the modulation introduced by a standing SAW with a wavelength $\lambda_{\mathrm{SAW}}=8 \mu \mathrm{m}$. (b), (c) The energy dispersion and spatial distribution of polariton condensates in the array without SAW, respectively. (d) Calculated energies and spatial distribution of condensates in a potential defined by the cyan solid lines. (e), (f) The same as (b) and (c) in the presence of a SAW. (g) Calculated energies and spatial distribution of condensates with SAW. The image was acquired by summing simulation frames over two SAW periods. Additional states induced by the SAW within the band gap of the static lattice are indicated by the blue horizontal arrow.

\section{CONCLUSIONS AND PERSPECTIVES}

In the previous sections, we have demonstrated dynamic control of confined polariton states in a structured MC using both optical and acoustic fields. The studies were carried out on a $\mathrm{MC}$ designed to be in the strong-coupling regime, both within the traps and in the potential barriers around them. This special configuration enables an efficient optical control of the confinement potential by changing the polariton density using light. In particular, we showed that the different energy shifts due to polariton-polariton interactions in the trap and in the surrounding barriers enable the variation of the confinement depth by as much as $200 \%$. Correspondingly, the strain of a few hundred megahertz SAW $\left(f_{\mathrm{SAW}}\right)$ can be used to modulate the energies of trapped polaritons. Depending on the ratio of the trap size $\left(w_{\text {trap }}\right)$ to the SAW wavelength $\left(\lambda_{\mathrm{SAW}}\right)$, we demonstrated three important acoustic functionalities: (i) $w_{\text {trap }}<\lambda_{\mathrm{SAW}}$, the modulation of the trap energies up to $1.5 \mathrm{meV}$ at $f_{\mathrm{SAW}}$; (ii) $w_{\text {trap }} \sim \lambda_{\mathrm{SAW}}$, the modulation occurs at $2 \times f_{\mathrm{SAW}}$, and the spatial localization of polariton wave functions is controlled via dynamic changes in the trap potential shape induced by the SAW; and (iii) when the separation between traps is approximately $\lambda_{\mathrm{SAW}} / 2$, the SAW brings the neighboring traps in and out of resonance at $f_{\mathrm{SAW}}$. Thus, the acoustic modulation enables new regimes where the energy, symmetry, and interactions of the trapped polaritons and condensates can be tuned independently of the particle density.

As an important proof of concept, we have demonstrated the acoustic modulation of the dispersion of an array of coupled static traps which gives rise to new dynamic coherent lattice states. The demonstrated results are very well reproduced by a numerical model for a particle in a box based on the realistic potential profile of a trap. The results clearly show that the modulation by SAWs has several important advantages: (i) a wide range of achievable amplitudes and phases of the acoustic modulation; (ii) due to the low acoustic absorption, SAWs propagate over several millimeters and thus the modulation can be extended to arbitrary length scales; (iii) the pure strain modulation can be used for manipulation of quantum polariton lattices.

The tunability arising from the acoustic modulation brings several advantages to intracavity traps. As was discussed recently in Ref. [45], the operation of lattices of interacting polariton condensates depends strongly on the weak local disorder. In this regard, one major concern for future applications of polariton lattices is (quantum) decoherence. Two relevant decoherence mechanisms are (i) the scattering by thermal phonons and (ii) potential disorder. The above considerations further highlight the advantages of using SAWs: (i) contrary to thermal vibrations, SAWs are stimulated coherent phonons with well-defined frequency and amplitude, and (ii) due to the weak propagation losses, the SAW amplitude remains constant over macroscopic, that is, millimeter sizes. This behavior is evidenced by the results in Fig. 3(b), where the modulation amplitude of the condensate energy in the ground state remains constant and sinusoidal over multiple SAW periods. Thus, the above two properties ensure the perfectly sinusoidal modulation across arbitrary large lattices without introducing quantum decoherence.

While the acoustic modulation (even in the case of a standing wave) is transient, it is possible to use it to realize quasistatic configurations of the polariton energies in lattices. The latter applies, in particular, for subgigahertz SAW frequencies, as in the present case. In fact, the acoustic period of $2.6 \mathrm{~ns}$ used here is at least 2 orders of magnitude larger than the coherence time of the condensate (about $100 \mathrm{ps).}$ Thus, any given realization of a condensate "sees" a "static" acoustic potential. One could take advantage of this by making stroboscopiclike experiment, by synchronizing the short (e.g., picosecond) laser pulses with a particular SAW phase.

Acoustic waves offer strategies for reaching dynamic control down to the single-polariton quantum regime, which 
normally cannot be achieved by purely optical control. This regime requires submicrometer confinement dimensions-a task that necessitates high-quality polariton traps with submicrometer dimensions in order to give rise to the on-site polariton interactions exceeding the particle spectral linewidths. We envision the following two strategies to dynamically control trapped polaritons in the quantum regime. The first approach relies on the use of SAWs with submicrometer wavelengths (and frequencies above $3 \mathrm{GHz}$ in GaAs structures, respectively) to create perfect (i.e., with negligible disorder) lattices consisting of traps with dimensions of $\sim \lambda_{\mathrm{SAW}} / 2$. The second strategy consists in the application of submicrometer SAWs to control on-site and intersite interactions in static lattices of traps with submicrometer periods. Since the penetration depth of the SAW fields is comparable to $\lambda_{\mathrm{SAW}}$, the main challenge for the above approaches will be to couple a high-frequency acoustic field to the MC active region (namely, the MC spacer with QWs) while keeping a high-optical-quality factor. Here, open MCs, where the monolithic upper distributed Bragg reflector (DBR) is replaced by one suspended above the cavity spacer [46-48], provide an interesting solution. SAWs can then be generated directly on the spacer region of the MC, thus leading to strong acoustic fields. Finally, GHz SAWs will push the modulation timescale below $300 \mathrm{ps,} \mathrm{making} \mathrm{it}$ comparable to the polariton coherence time, allowing one to explore nonadiabatic dynamics of confined polaritons, e.g., tunable polariton Floquet lattices [36]. The proposed tunable platform is thus an important step towards novel solid-state photonic devices such as classical and quantum simulators, qubits, and topologically protected photonic on-chip circuitry.

\section{ACKNOWLEDGMENTS}

We are grateful to J. Lähnemann (PDI) for discussions and for a critical review of the manuscript. We also acknowledge the financial support from the German DFG (Grant No. 359162958).

\section{APPENDIX A: SAMPLE FABRICATION AND EXPERIMENTAL METHODS}

\section{Sample structure}

The studies were carried out on an (Al,Ga)As MC grown on a 2-inch GaAs (001) substrate by molecular beam epitaxy (MBE). The 4.43- $\mu$ m-thick lower DBR consists of $36 \times$ $\lambda / 4$ ( $\lambda$ is the optical wavelength in the material) stacks of $\mathrm{Al}_{0.15} \mathrm{Ga}_{0.85} \mathrm{As} / \mathrm{Al}_{x} \mathrm{Ga}_{1-x}$ As layer pairs with an $\mathrm{Al}$ composition $x$ continuously reducing from 0.80 in the first stack (i.e., the one closest to the substrate) to 0.45 in the last stack. The $0.448-\mu$ m-thick $3 \times \lambda / 2 \mathrm{Al}_{0.30} \mathrm{Ga}_{0.70}$ As $\mathrm{MC}$ spacer on top of the lower DBR contains three pairs of 15-nm-thick GaAs QWs placed at the antinode positions of the MC optical mode. After the deposition of a 120 -nm-thick $\mathrm{Al}_{0.15} \mathrm{Ga}_{0.85} \mathrm{As}$ layer on the uppermost QW structure, the sample was taken out of the MBE chamber and patterned by means of photolithography and wet chemical etching to form 12-nm-high mesas with different shapes. The location of the etched layer was chosen to coincide with a node of the optical cavity mode of the final structure. In this way, one minimizes the impact of surface defects introduced by the etching on the
MC properties. The sample was then reintroduced into the MBE for the overgrowth of the remaining part of the spacer and of the 2.49- $\mu \mathrm{m}$-thick upper DBR, which consists of $20 \times$ $\lambda / 4$ pairs of $\mathrm{Al}_{0.15} \mathrm{Ga}_{0.85} \mathrm{As} / \mathrm{Al}_{0.75} \mathrm{Ga}_{0.25}$ As layer stacks. The spacer etching results in a blueshift of the cavity optical mode by $9 \mathrm{meV}$. Basic structural and optical properties of the studied cavity were reported in Ref. [32]. There, we have shown that the strong photon-exciton coupling regime is achieved in both etched and nonetched regions, resulting in polariton states with different exciton-to-photon contents. The energy difference between the lower polariton of the etched region (ER) and that of the no-etched region (nER) defines the barrier energy, with nonetched mesas acting as traps.

\section{Spectroscopic studies}

The spectroscopic studies of the acoustic modulation were performed on intracavity polariton traps formed by the overgrowth of square mesas with nominal dimensions of $1.6 \times$ $1.6 \mu \mathrm{m}^{2}$ and $4 \times 4 \mu \mathrm{m}^{2}$, a $10-\mu \mathrm{m}$-wide and $200-\mu \mathrm{m}$-long wire mesa, and an array of 1D 4.5- $\mu \mathrm{m}$-wide wires with period of $5 \mu \mathrm{m}$. The time-integrated emission from the confined states was probed by low-temperature photoluminescence (PL) at $10 \mathrm{~K}$ excited with a laser beam (energies of 1.6 or $1.63 \mathrm{eV}$ ) from a tunable Sirah Matisse Ti-sapphire cw laser. The laser was focused on the MC surface under near-normal incidence into Gaussian spots with diameters of 60 and $18 \mu \mathrm{m}$ for the excitation of polaritons in the linear and condensation regimes, respectively. The PL was spectrally dispersed by a single grating spectrometer (Princeton Instruments Acton SP2500) and detected by a liquid-nitrogen-cooled CCD camera (Princeton Instruments). The setup provides the spectral resolution of $0.085 \mathrm{meV}$. Two-dimensional (2D) images displaying the spectral PL distribution as a function of position on the sample surface were obtained by recording CCD frames along the cross section of the sample image projected on the spectrometer slit. Time-resolved measurements were carried out using an Optronis SC-10 streak camera with time resolution of $15 \mathrm{ps}$ synchronized with the ac voltage driving the IDTs.

\section{APPENDIX B: EXCITATION OF STANDING SURFACE ACOUSTIC WAVES}

\section{Acoustic delay line}

A standing SAW field was excited by applying a rf signal to one of the IDTs $\left(\mathrm{IDT}_{1}\right)$ and using the second, unpowered IDT $\left(\mathrm{IDT}_{2}\right)$ as an acoustic reflector, Fig. 2(b). The formation of a standing SAW field within the delay line can be inferred from measurements of the rf-reflection parameter $s_{11}$ for $\mathrm{IDT}_{1}$ displayed in Fig. 2(c), which was recorded at $10 \mathrm{~K}$ using a vector network analyzer. The $s_{11}$ spectrum shows a series of narrow resonances within the emission band of the IDT covering the frequency range from 381 to $386 \mathrm{MHz}$. These narrow resonances are attributed to the excitation of longitudinal SAW modes of the acoustic cavity defined by the IDTs. The corresponding time-domain profile for $s_{11}$ in Fig. 2(d) is characterized by a series of echoes separated by $t_{\text {Refl }} \approx$ $1.63 \mu \mathrm{s}$ intervals. The latter result from multiple round trips in the acoustic cavity defined by the spacing between the IDTs. Indeed, the echo delay $t_{\text {Refl }}$ is related to the effective round-trip 
distance $d_{\text {SAW-travel }}$ as $d_{\text {SAW-travel }}=v_{\text {SAW }} \times t_{\text {Refl }}=4890 \mu \mathrm{m}$, where $v_{\mathrm{SAW}}=3 \mu \mathrm{m} / \mathrm{ns}$ is the SAW velocity calculated using the elastic model for the MC (see Appendix B 2). The $d_{\text {SAW-travel }}$ is larger than twice the distance $d_{\text {IDTs }}=2000 \mu \mathrm{m}$ between the IDTs due to the penetration depth of the acoustic field in the IDT reflectors of approximately $450 \mu \mathrm{m}$.

Since the acoustic propagation losses in the $(\mathrm{Al}, \mathrm{Ga}) \mathrm{As}$ layer structure are expected to be small, the power ratio $R_{\mathrm{IDT}}$ between successive echoes in Fig. 2(d) becomes determined by the finite acoustic reflection coefficient of the IDTs. The measured ratio given by $R_{\mathrm{IDT}} \approx 4 \mathrm{~dB}$ ( 0.43 in linear scale) can then be used to determine the standing-wave ratio $\mathrm{SWR}=$ $\left(1+R_{\mathrm{IDT}}\right) /\left(1-R_{\mathrm{IDT}}\right)=2.5$, corresponding to the ratio between the maximum and minimum amplitudes of the SAW field.

The spectroscopy studies were performed by exciting IDT $_{1}$ at the narrow resonance at $f_{\mathrm{SAW}}=383.69 \mathrm{MHz}$ marked by the red arrow in Fig. 2(c). From the depth of this $s_{11}$ resonance, we estimate that approximately $P_{\text {coupled }}=0.5 \%$ of the incoming rf power is converted to acoustic modes irradiating from both sides of IDT $_{1}$. The SAWs were excited with nominal $\mathrm{rf}$ powers of up to $P_{\mathrm{rf}}=35 \mathrm{dBm}$, thus leading to the maximum linear power densities $P_{1}=P_{\text {coupled }} \times 10^{(\operatorname{Prf}-30) / 10} / w_{\text {SAW }}=$ $50 \mathrm{~W} / \mathrm{m}$, where $w_{\text {SAW }}=320 \mu \mathrm{m}$ is the IDT aperture.

\section{Modulation of energies by the surface acoustic waves}

The acoustic wavelength $\lambda_{\text {SAW }}=8 \mu \mathrm{m}$ was selected to maximize the strain-induced band-gap modulation at the depth of the QWs. Figure 7(a) displays the depth profile of the acoustic power density for the SAW with $\lambda_{\mathrm{SAW}}=8 \mu \mathrm{m}$, propagating on the microcavity surface. The calculations were carried out using an elastic model for the MC following the procedure outlined in Ref. [49]. The results in Fig. 7(a) were obtained assuming a SAW with a linear power density of $P_{1}=53 \mathrm{~W} / \mathrm{m}$. Here, $P_{1}$ is defined as the ratio between the total SAW power flux and the width of the acoustic beam, which is approximately equal to the IDT aperture $(320 \mu \mathrm{m}$, in the present case). If one takes into account the acoustoelectric conversion estimated in Appendix B 1, this power density approximately corresponds to the maximum SAW intensities used in the spectroscopic experiments.

The elastic model yields the spatial distribution of the SAW strain $\left(\varepsilon_{\mathrm{ij}}\right)$ and piezoelectric field in the MC structures. The calculated strain field was then combined with the deformation potentials for GaAs to determine the spatial modulation of the QW energy levels. Figure 7(b) compares profiles for the spatial modulation of the electron-heavy hole $\left(\Delta E_{\mathrm{e}-\mathrm{hh}}\right)$ and electron-light hole $\left(\Delta E_{\mathrm{e}-\mathrm{lh}}\right)$ transitions of the QWs embedded in the MC spacer. While the e-lh transition is only slightly affected by the SAW strain, the e-hh transition energy oscillates with an amplitude of approximately $\pm 0.8 \mathrm{meV}$ around the unperturbed band-gap energy. Figure 7(b) also displays the calculated modulation of the optical MC resonance energy $\left(\Delta E_{c}\right)$. The latter was determined by taking into account the modulation of the refractive index of the MC spacer using the GaAs acousto-optic coefficients, as well as the modulation of the spacer thickness induced by the SAW strain (see Refs. [44,49] for details). The profiles of Fig. 7(b) show that the main mechanism of the polariton modulation is the strain- (a)
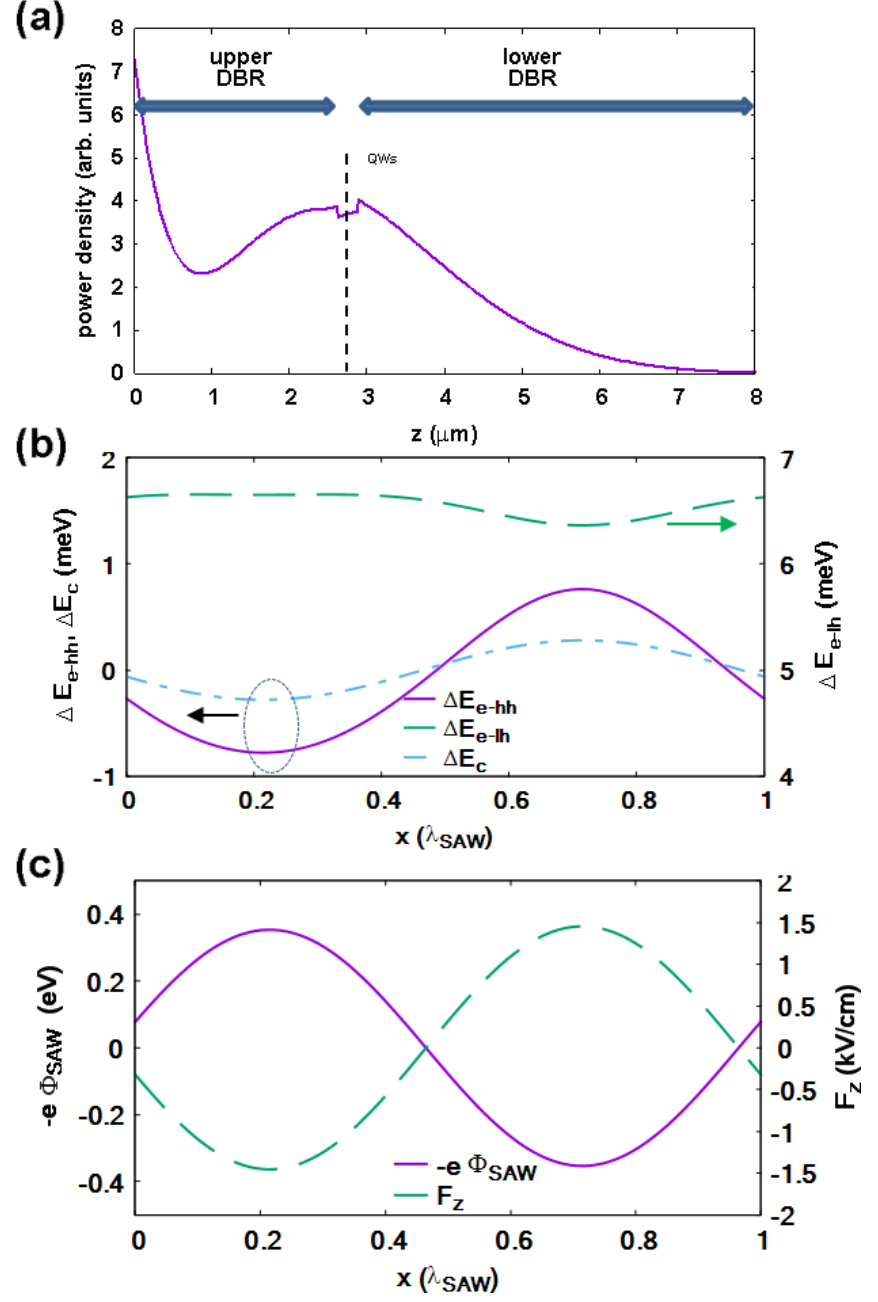

FIG. 7. (a) Calculated depth profile of the acoustic power density for a SAW with wavelength $\lambda_{\text {SAW }}=8 \mu \mathrm{m}$ propagating along the $x \|[-110]$ direction of the microcavity surface. (b) Spatial dependence of the strain-induced energy modulation of the MC photonic resonance $\left(\Delta E_{c}\right)$ as well as of the deformation-potential modulation of the electron-heavy hole $\left(\Delta E_{\mathrm{e}-\mathrm{hh}}\right)$ and electron-light hole $\left(\Delta E_{\mathrm{e}-\mathrm{lh}}\right)$ QW transitions. (c) Spatial dependence of the electronic piezoelectric energy $\left(-e \Phi_{\mathrm{SAW}}\right)$ and of the vertical component of the SAW piezoelectric field $\left(F_{z}\right)$. The calculations were carried out using an elastic model for the MC and assuming a SAW linear power density of $P_{1}=53 \mathrm{~W} / \mathrm{m}$. ( $P_{1}$ is defined as the ratio between the SAW power and the width of the acoustic beam.) $\Delta E_{c}$ includes the modulation of the refractive index as well as the thickness of the layers by the SAW strain.

induced changes of the band gap of the QWs, resulting in the modulation of the QW exciton energy. The modifications of the MC photon energy due to the strain-induced changes of the spacer thickness and refractive index account only for about $30 \%$ of the total modulation [43]. The modulation of the excitonic and photonic energies are used in Appendix D to determine the modulation of polariton levels under SAW excitation.

In contrast to previous investigations of polariton modulation by SAWs in unstructured MCs [29], the present studies were carried out using piezoelectric SAWs; their strain fields 


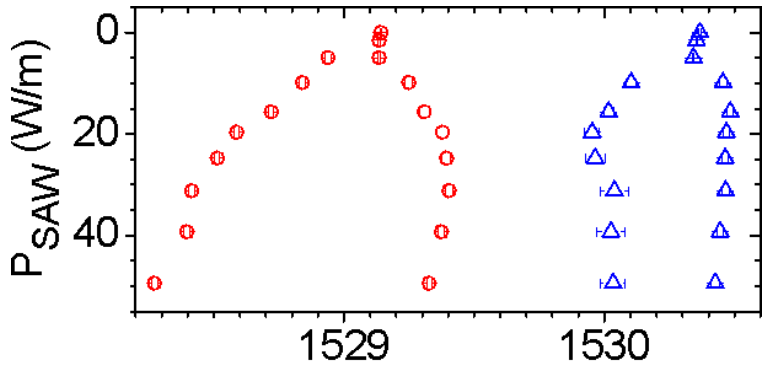

(a)

Energy (meV)

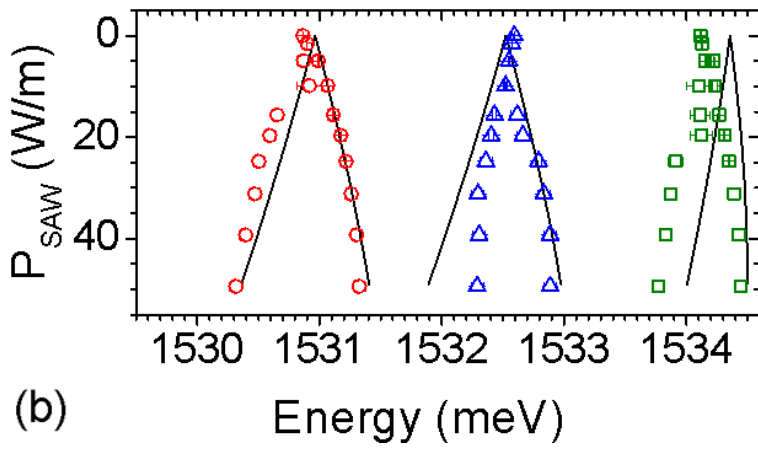

FIG. 8. Dependence of the energies of the side peaks of the SAW-split energy levels $(n=1,2,3)$ of the trap on the SAW power recorded for optical excitation of (a) $0.001 \times P_{t h}$ and (b) $3 \times P_{t h}$. These energies were obtained by fitting the spatially integrated spectra of Figs. 1(c) and 1(d) to multiple Gaussian lines. The solid black lines in (b) represent calculated splitting.

are accompanied by electric fields oriented along the $x\left(F_{x}\right)$ and $z\left(F_{z}\right)$ directions. The spatial dependence of the vertical component $F_{z}$ as well as the electronic piezoelectric energy $-e \Phi_{\mathrm{SAW}}$ induced by $F_{x}$ are displayed in Fig. 7(c).

\section{APPENDIX C: ACOUSTIC MODULATION AT LOW POLARITON DENSITIES}

We consider modulation effects in the linear polariton regime (i.e., at low excitation densities). The PL emission intensity under these conditions becomes strongly suppressed at high acoustic excitation [compare Figs. 1(a) and 1(e)] due to ionization of excitons by the piezoelectric field. The lower emission intensity makes it difficult to follow the temporal evolution of the confined energy levels using our streak camera setup. The results from Sec. II B 1 [cf. Fig. 1(c)] show, however, that the amplitude of the SAW-induced energy modulation can be directly extracted from the energy splitting of the doublets observed in the time-integrated PL profiles shown in Figs. 1(c) and 1(d). Figures 8(a) and 8(b) compare the dependence on SAW power of the energies of the doublet peaks measured in the linear and condensed regime, respectively. For a given SAW amplitude, the energy splittings are larger in the linear regime than for condensates. A similar behavior has been observed for the modulation amplitude of
SAW-induced polariton lattices, attributed to the screening of the SAW modulation potential at high polariton densities [50].

In the condensed regime, the strain-induced splitting for all confined levels is symmetric with respect to the unperturbed trap energies (i.e., in the absence of the SAW excitation). The numerical model of Appendix D reproduces the symmetrical splittings as well as their magnitudes for comparable acoustic powers, as shown by the solid lines in Fig. 8(b). The measured splittings in the linear regime, in contrast, are symmetric only for acoustic powers up to $P_{\mathrm{SAW}} \approx 10 \mathrm{~W} / \mathrm{m}$ [cf. Fig. 8(a)]. For higher powers, the splitting continues to increase but is now accompanied by a redshift of both components. A possible mechanism for the redshift is the quantum confined Stark effect induced by the vertical component $F_{z}$ of the SAW piezoelectric field [cf. Fig. 7(c)]. $F_{z}$ is expected to induce a redshift estimated to be up to $\sim 1 / 2 F_{z} d_{\mathrm{QW}} \sim 1 \mathrm{meV}$ at the antinodes of the strain field, which compares well to the values observed in Fig. 8(a).

\section{APPENDIX D: NUMERICAL MODEL FOR THE STRAIN-INDUCED MODULATION OF THE CONFINED LEVELS}

The polariton confinement in the intracavity traps is due to the lateral modulation of the optical resonance energies of the MC. As discussed in the Appendix B 2, the SAW strain and piezoelectric fields dynamically modulate the bare excitonic and photonic energies of the MC [cf. Fig. 7(b)]. We will neglect, for the moment, the impact of the piezoelectric field on the polariton energies; this assumption is expected to be a good approximation for the condensate regime, where the field becomes screened by the high polariton densities. The latter is evidenced by almost identical total intensities of the curves in Fig. 3(a).

The effects of the SAW strain field on confined polaritons can be taken into account by assuming that excitons and photons respond adiabatically to the acoustic field. At each spatial coordinate $(\vec{r}$, measured relative to the center of the trap), the energy of the bare cavity photons (index $i=c)$, electron-heavy hole $(i=\mathrm{e}-\mathrm{hh})$, and electron-light hole $(i=\mathrm{e}-\mathrm{lh})$ excitons can thus be expressed as $E_{i}(\vec{r})+$ $\Delta E_{i}\left(\vec{r}, \varphi_{\mathrm{SAW}}\right)$, where $\varphi_{\mathrm{SAW}}$ is the SAW phase. The first terms $E_{i}(\vec{r})$ denote the unperturbed bare energies in the structured MC - these terms are independent of $\vec{r}$ for the excitonic levels, which are not affected by the variations in spacer thickness. The second term can be expressed as $\Delta E_{i}\left(\vec{r}, \varphi_{\mathrm{SAW}}\right)=$ $\Delta E_{i, 0} \cos \left(\vec{k}_{\mathrm{SAW}} \cdot \vec{r}\right) \cos \left(\varphi_{\mathrm{SAW}}\right)$, where $\Delta E_{i, 0}$ is the corresponding modulation amplitudes, and $\vec{k}_{\mathrm{SAW}}=\frac{2 \pi}{\lambda_{\mathrm{SAW}}} \hat{x}$ the SAW wave vector. Like in the experiments, the phase $\varphi_{\mathrm{SAW}}$ of the standing SAW was chosen to have an antinode at the center of the trap corresponding to the position $r=0$. The polariton energies can be determined by including the Rabi splittings $\Omega_{e-h h}(\vec{r})$ and $\Omega_{e-l h}(\vec{r})$ for the electron-heavy hole and electron-light hole transitions, respectively, and diagonalizing the following Hamiltonian:

$$
H_{p}\left(\vec{r}, \varphi_{\mathrm{SAW}}\right)=\left(\begin{array}{ccc}
E_{c}(\vec{r})+\Delta E_{c}\left(\vec{r}, \varphi_{\mathrm{SAW}}\right) & \frac{\Omega_{e-h h}(\vec{r})}{2} & \frac{\Omega_{e-l h l}(\vec{r})}{2} \\
\frac{\Omega_{e-h h}(\vec{r})}{2} & E_{e-h h}-\Delta E_{e-h h}\left(\vec{r}, \varphi_{\mathrm{SAW}}\right) & 0 \\
\frac{\Omega_{e-l h}(\vec{r})}{2} & 0 & E_{e-l h}+\Delta E_{e-l h}\left(\vec{r}, \varphi_{\mathrm{SAW}}\right)
\end{array}\right)
$$


TABLE I. Sample parameters used in the calculations (based on Ref. [32]).

\begin{tabular}{lcl}
\hline \hline Parameter & Value & \\
\hline$E_{\mathrm{e}-\mathrm{hh}}(\mathrm{meV})$ & 1534.8 & Description \\
$E_{\mathrm{e}-\mathrm{hh}}(\mathrm{meV})$ & 1541 & Light-hole energy \\
$E_{c, \min }(\mathrm{meV})$ & 1530.8 & Lowest bare cavity energy \\
$E_{c, \max }(\mathrm{meV})$ & 1541.5 & Highest bare cavity energy \\
$\Omega_{\mathrm{e}-\mathrm{hh}}(\mathrm{meV}) \mathrm{nER} / \mathrm{ER}$ & $7.2 / 6$ & Rabi splitting for electron-heavy hole transition in the nonetched (nER) and etched (ER) regions \\
$\Omega_{\mathrm{e}-\mathrm{lh}}(\mathrm{meV}) \mathrm{nER} / \mathrm{ER}$ & $5.5 / 4.5$ & Rabi splitting for electron-light hole transition in nER and ER \\
$m_{p}\left(m_{e}\right)$ & $5.5 \times 10^{-5}$ & Polariton effective mass in free electron mass \\
\hline \hline
\end{tabular}

Here $E_{c}(\vec{r})$ represents the spatial dependence of the bare photonic energy induced by the structuring of the MC spacer layer; it was determined from spatial profiles of the trap surface relief reported in Ref. [32]. As was discussed in that work, the cavity quality factor is approximately $20 \%$ higher in the nonetched regions than in the etched ones. The latter was taken into account by assuming a $20 \%$ larger Rabi splitting in the nonetched areas. The SAW modulation parameters used in the calculations were taken from Fig. 7(b). The weaker acoustic modulation of the electron-light hole transitions was taken into account by assuming an amplitude ratio $\frac{\Delta E_{e-h h, 0}}{\Delta E_{\mathrm{e}-\mathrm{h}, 0}}=$ -3 . The remaining parameters, which are summarized in Table I, were extracted from [32].

To determine the confined polariton levels under acoustic excitation, we assumed that the polaritons experience an effective modulation potential given by the spatial dependence of the lowest energy eigenvalue of Eq. (1). The confined levels were determined by numerically solving the Schrödinger equation for this potential assuming a polariton effective mass $m_{\text {eff }}=5.5 \times 10^{-5} m_{e}$, where $m_{e}$ denotes the free electron mass. At low particle density (low optical pump), the above model predicts the energy spectrum and the spatial extent of the polariton wave functions of the trap very accurately, as shown in Fig. 1(a). Here, the dashed and solid white lines represent the calculated trap potential and wave functions of the confined levels, respectively.

Above the condensation threshold, polariton-polariton interactions as well as polariton interactions with the excitonic reservoir created by the nonresonant optical pump are expected to blueshift the polariton energies by several millielectronvolts [51]. These interaction effects were modeled by increasing the energies of the barrier and the trap bottom by $\Delta E_{\mathrm{Barrier}}=6.6 \mathrm{meV}$ and $\Delta E_{\text {Trap }}=1.55 \mathrm{meV}$, respectively. The barrier energy shift was determined from experimental data of Fig. 1(b), which shows that the barrier of the trap is blueshifted by approximately $\Delta E_{\text {Barrier }} \approx 6 \pm 0.6 \mathrm{meV}$ under high optical excitation densities [cf. red arrows in Figs. 1(a) and $1(\mathrm{~b})]$. The energy shift at the trap was selected to satisfy a ratio $\Delta E_{\text {Barrier }} / \Delta E_{\text {Trap }}=4.3$, equal to the ratio between the excitonic Hopfield coefficients for polaritons in the etched and unetched areas of the structured MC [32].

In the case of the $10-\mu \mathrm{m}$-wide wire, the same model gives the best match to the experiment assuming $\Delta E_{\text {Barrier }}=6.6 \mathrm{meV}$ and $\Delta E_{\text {Trap }}=1.45 \mathrm{meV}$, cf. Figs. 4(a) and 4(b).

\section{APPENDIX E: TIME-DEPENDENT INTENSITY MODULATION}

The energy modulation of the condensate energies is accompanied by a modulation of the PL intensity at the SAW frequency, which is very pronounced for small traps [cf. Fig. 3(b)]. In particular, the PL intensity increases by up to 1 order of magnitude when the level energies are blueshifted by the acoustic field. We note here that this change in intensity is still at least 3 orders of magnitude smaller than that due to the condensation. In the nonetched regions of the MC (corresponding to the trap site), the polariton states are negatively detuned (i.e., they have a predominantly photonic character). A possible mechanism for the intensity modulation is the change in the effective detuning of the confined mode as its energy is changed by the SAW field. In the present case, however, the SAW-induced blueshift is less than $20 \%$ of the Rabi splitting so that this mechanism alone cannot account for the intensity modulation. Furthermore, since the modulation amplitude of the e-hh transition is larger than that of the photonic mode [cf. Fig. 7(b)], the detuning becomes more negative as the strain blueshifts the e-hh level. Thus, the coupling of the confined levels to light should reduce, which should result in the decrease of the PL intensity. In contrast, the latter increases.

A more likely mechanism for the PL intensity modulation is associated with the SAW piezoelectric potential. In the present experiments, polaritons are excited nonresonantly via the energy relaxation of excitons, which can dissociate under the piezoelectric field. The resulting free electrons and holes can then leave the trap since they do not "feel" the optical potential responsible for polariton confinement. As discussed in detail in Ref. [42], the exciton dissociation probability is minimized for the SAW phases corresponding to the minimum of the piezoelectric energy $-e \Phi_{\mathrm{SAW}}$. In the present case, these phases also correspond to the phases of maximum band-gap blueshift, as can be inferred from the profiles in Fig. 7. This behavior results from the different transport properties for electrons and holes in the type-II piezoelectric potential produced by the SAW [cf. Fig. 7(c)]. If an exciton dissociates during a SAW phase close to the minimum of $-e \Phi_{\mathrm{SAW}}$, the hole remains trapped while the electron drifts away. Close to the maximum of $-e \Phi_{\mathrm{SAW}}$, in contrast, the electron remains trapped while the holes drift away. Exciton dissociation is, however, more probable in the first case, since more mobile electrons can more efficiently move away from the hole. As 
a result, one expects to have a higher exciton density close to the minima of $-e \Phi_{\mathrm{SAW}}$, which coincides with the phases of highest cavity and electron-heavy hole transition energies [cf. Figs. 7(b) and 7(c)]. Similar effects are expected to be responsible for the intensity modulation of the wire PL, shown in Fig. 4(e).
[1] I. Carusotto and C. Ciuti, Quantum fluids of light, Rev. Mod. Phys. 85, 299 (2013).

[2] J. Kasprzak et al., Bose-Einstein condensation of exciton polaritons, Nature (London) 443, 409 (2006).

[3] V. Savona, C. Piermarocchi, A. Quattropani, P. Schwendimann, and F. Tassone, Optical properties of microcavity polaritons, Phase Transitions 68, 169 (1999).

[4] C. Weisbuch, M. Nishioka, A. Ishikawa, and Y. Arakawa, Observation of the Coupled Exciton-Photon Mode Splitting in a Semiconductor Quantum Microcavity, Phys. Rev. Lett. 69, 3314 (1992).

[5] R. Butté, G. Delalleau, A. I. Tartakovskii, M. S. Skolnick, V. N. Astratove, J. J. Baumberg, G. Malpuech, A. Di Carlo, A. V. Kovokin, and J. S. Roberts, Transition from strong to weak coupling and the onset of lasing in semiconductor microcavities, Phys. Rev. B 65, 205310 (2002).

[6] W. Gregor, H. Deng, R. Huang, M. Sugita, F. Tassone, and Y. Yamamoto, Exciton-polariton lasing in a microcavity, Semicond. Sci. Technol. 18, S386 (2003).

[7] D. Porras, C. Ciuti, J. J. Baumberg, and C. Tejedor, Polariton dynamics and Bose-Einstein condensation in semiconductor microcavities, Phys. Rev. B 66, 085304 (2002).

[8] P. Senellart and J. Bloch, Nonlinear Emission of Microcavity Polaritons in the Low Density Regime, Phys. Rev. Lett. 82, 1233 (1999).

[9] R. M. Stevenson, V. N. Astratov, M. S. Skolnick, D. M. Whittaker, M. Emam-Ismail, A. I. Tartakovskii, P. G. Savvidis, J. J. Baumberg, and J. S. Roberts, Continuous Wave Observation of Massive Polariton Redistribution by Stimulated Scattering in Semiconductor Microcavities, Phys. Rev. Lett. 85, 3680 (2000).

[10] A. I. Tartakovskii, M. Emam-Ismail, R. M. Stevenson, M. S. Skolnick, V. N. Astratov, D. M. Whittaker, J. J. Baumberg, and J. S. Roberts, Relaxation bottleneck and its suppression in semiconductor microcavities, Phys. Rev. B 62, R2283 (2000).

[11] P. St-Jean, V. Goblot, E. Galopin, A. Lemaître, T. Ozawa, L. Le Gratiet, I. Sagnes, J. Bloch, and A. Amo, Lasing in topological edge states of a one-dimensional lattice, Nat. Photonics 11, 651 (2017).

[12] K. G. Lagoudakis, B. Pietka, M. Wouters, R. André, and B. Deveaud-Plédran, Coherent Oscillations in an ExcitonPolariton Josephson Junction, Phys. Rev. Lett. 105, 120403 (2010).

[13] A. Amo, J. Lefrère, S. Pigeon, C. Adrados, C. Ciuti, I. Carusotto, R. Houdré, E. Giacobino, and A. Bramati, Superfluidity of polaritons in semiconductor microcavities, Nat. Phys. 5, 805 (2009)

[14] F. Marsault, H. S. Nguyen, D. Tanese, A. Lemaître, E. Galopin, I. Sagnes, A. Amo, and J. Bloch, Realization of an all optical exciton-polariton router, Appl. Phys. Lett. 107, 201115 (2015).

[15] G. Grosso, S. Trebaol, M. Wouters, F. Morier-Genoud, M. T. Portella-Oberli, and B. Deveaud, Nonlinear relaxation and selective polychromatic lasing of confined polaritons, Phys. Rev. B 90, 045307 (2014).

[16] D. Sanvitto and S. Kéna-cohen, The road towards polaritonic devices, Nat. Mater. 15, 1061 (2016).

[17] S. S. Demirchyan, I. Y. Chestnov, A. P. Alodjants, M. M. Glazov, and A. V. Kavokin, Qubits Based on Polariton Rabi Oscillators, Phys. Rev. Lett. 112, 196403 (2014).

[18] T. Karzig, C.-E. Bardyn, N. H. Lindner, and G. Refael, Topological Polaritons, Phys. Rev. X 5, 031001 (2015).

[19] S. Klembt, T. H. Harder, O. A. Egorov, K. Winkler, R. Ge, M. A. Bandres, M. Emmerling, L. Worschech, T. C. H. Liew, M. Segev, C. Schneider, and S. Höfling, Excitonpolariton topological insulator, Nature (London) 562, 552 (2018).

[20] A. Amo and J. Bloch, Exciton-polaritons in lattices: A nonlinear photonic simulator, C. R. Phys. 17, 934 (2016).

[21] N. G. Berloff, M. Silva, K. Kalinin, A. Askitopoulos, J. D Töpfer, P. Cilibrizzi, W. Langbein, and P. G. Lagoudakis, Realizing the classical XY Hamiltonian in polariton simulators, Nat. Mater. 16, 1120 (2017).

[22] N. Y. Kim, K. Kusudo, C. Wu, N. Masumoto, A. Löffler, S. Höfling, N. Kumada, L. Worschech, A. Forchel, and Y. Yamamoto, Dynamical $d$-wave condensation of excitonpolaritons in a two-dimensional square-lattice potential, Nat. Phys. 7, 681 (2011).

[23] N. Masumoto, N. Y. Kim, T. Byrnes, K. Kusudo, A. Löffler, S. Höfling, A. Forchel, and Y. Yamamoto, Exciton-polariton condensates with flat bands in a two-dimensional kagome lattice, New J. Phys. 14, 065002 (2012).

[24] E. A. Cerda-Méndez, D. Sarkar, D. N. Krizhanovskii, S. S Gavrilov, K. Biermann, M. S. Skolnick, and P. V. Santos, Exciton-Polariton Gap Solitons in Two-Dimensional Lattices, Phys. Rev. Lett. 111, 146401 (2013)

[25] G. Dasbach, M. Schwab, M. Bayer, D. N. Krizhanovskii, and A. Forchel, Tailoring the polariton dispersion by optical confinement: Access to a manifold of elastic polariton pair scattering channels, Phys. Rev. B 66, 201201(R) (2002).

[26] D. Bajoni, P. Senellart, E. Wertz, I. Sagnes, A. Miard, A. Lemaître, and J. Bloch, Polariton Laser Using Single Micropillar GaAs-GaAlAs Semiconductor Cavities, Phys. Rev. Lett. 100, 047401 (2008).

[27] L. Ferrier, E. Wertz, R. Johne, D. D. Solnyshkov, P. Senellart, I. Sagnes, A. Lemaître, G. Malpuech, and J. Bloch, Interactions in Confined Polariton Condensates, Phys. Rev. Lett. 106, 126401 (2011).

[28] A. Baas, O. El Daïf, M. Richard, J.-P. Brantut, G. Nardin, R. Idrissi Kaitouni, T. Guillet, V. Savona, J. L. Staehli, F. MorierGenoud, and B. Deveaud, Zero dimensional exciton-polaritons, Phys. Status Solidi 243, 2311 (2006).

[29] E. A. Cerda-Méndez, D. N. Krizhanovskii, M. Wouters, R. Bradley, K. Biermann, K. Guda, R. Hey, P. V. Santos, D. Sarkar, and M. S. Skolnick, Polariton Condensation in Dynamic Acoustic Lattices, Phys. Rev. Lett. 105, 116402 (2010). 
[30] A. Askitopoulos, H. Ohadi, A. V. Kavokin, Z. Hatzopoulos, P. G. Savvidis, and P. G. Lagoudakis, Polariton condensation in an optically induced two-dimensional potential, Phys. Rev. B 88, 041308(R) (2013).

[31] J. Schmutzler, P. Lewandowski, M. Aßmann, D. Niemietz, S. Schumacher, M. Kamp, C. Schneider, S. Höfling, and M. Bayer, All-optical flow control of a polariton condensate using nonresonant excitation, Phys. Rev. B 91, 195308 (2015).

[32] A. S. Kuznetsov, P. L. J. Helgers, K. Biermann, and P. V. Santos, Quantum confinement of exciton-polaritons in a structured (Al,Ga)As microcavity, Phys. Rev. B 97, 195309 (2018).

[33] P. G. Lagoudakis and N. G. Berloff, A polariton graph simulator, New J. Phys. 19, 125008 (2017).

[34] M. Metcalfe, S. M. Carr, A. Muller, G. S. Solomon, and J. Lawall, Resolved Sideband Emission of InAs/GaAs Quantum Dots Strained by Surface Acoustic Waves, Phys. Rev. Lett. 105, 037401 (2010).

[35] D. A. Golter, T. Oo, M. Amezcua, I. Lekavicius, K. A. Stewart, and H. Wang, Coupling a Surface Acoustic Wave to an Electron Spin in Diamond Via a Dark State, Phys. Rev. X 6, 041060 (2016).

[36] A. Eckardt, Colloquium: Atomic quantum gases in periodically driven optical lattices, Rev. Mod. Phys. 89, 011004 (2017).

[37] H. Lignier, C. Sias, D. Ciampini, Y. Singh, A. Zenesini, O. Morsch, and E. Arimondo, Dynamical Control of Matter-Wave Tunneling in Periodic Potentials, Phys. Rev. Lett. 99, 220403 (2007).

[38] V. V. Ivanov, A. Alberti, M. Schioppo, G. Ferrari, M. Artoni, M. L. Chiofalo, and G. M. Tino, Coherent Delocalization of Atomic Wave Packets in Driven Lattice Potentials, Phys. Rev. Lett. 100, 043602 (2008).

[39] C. Rocke, S. Zimmermann, A. Wixforth, J. P. Kotthaus, G. Böhm, and G. Weimann, Acoustically Driven Storage of Light in a Quantum Well, Phys. Rev. Lett. 78, 4099 (1997).

[40] P. V. Santos, M. Ramsteiner, and F. Jungnickel, Spatially resolved photoluminescence in GaAs surface acoustic wave structures, Appl. Phys. Lett. 72, 2099 (1998).

[41] T. Sogawa, P. V. Santos, S. K. Zhang, S. Eshlaghi, A. D. Wieck, and K. H. Ploog, Dynamic band-structure modulation of quantum wells by surface acoustic waves, Phys. Rev. B 63, 121307(R) (2001).

[42] F. Alsina, P. V. Santos, and R. Hey, Spatial-dispersion-induced acoustic anisotropy in semiconductor structures, Phys. Rev. B 65, 193301 (2002).

[43] E. A. Cerda-Méndez, D. Krizhanovskii, K. Biermann, R. Hey, P. V. Santos, and M. S. Skolnick, Effects of the piezoelectric field on the modulation of exciton polaritons by surface acoustic waves, Superlattices Microstruct. 49, 233 (2011).

[44] M. M. de Lima, M. van der Poel, P. V. Santos, and J. M. Hvam, Phonon-Induced Polariton Superlattices, Phys. Rev. Lett. 97, 045501 (2006).

[45] H. Ohadi, Y. del Valle-Inclan Redondo, A. J. Ramsay, Z. Hatzopoulos, T. C. H. Liew, P. R. Eastham, P. G. Savvidis, and J. J. Baumberg, Synchronization crossover of polariton condensates in weakly disordered lattices, Phys. Rev. B 97, 195109 (2018).

[46] P. R. Dolan, G. M. Hughes, F. Grazioso, B. R. Patton, and J. M. Smith, Femtoliter tunable optical cavity arrays, Opt. Lett. 35, 3556 (2010).

[47] S. Dufferwiel et al., Strong exciton-photon coupling in open semiconductor microcavities, Appl. Phys. Lett. 104, 192107 (2014).

[48] B. Besga, C. Vaneph, J. Reichel, J. Estève, A. Reinhard, J. Miguel-Sánchez, A. Imamoglu, and T. Volz, Polariton Boxes in a Tunable Fiber Cavity, Phys. Rev. Appl. 3, 014008 (2015).

[49] M. de Lima Jr. and P. V. Santos, Modulation of photonic structures by surface acoustic, Rep. Prog. Phys. 68, 1639 (2005).

[50] D. N. Krizhanovskii, E. A. Cerda-Méndez, S. Gavrilov, D. Sarkar, K. Guda, R. Bradley, P. V. Santos, R. Hey, K. Biermann, M. Sich, F. Fras, and M. S. Skolnick, Effect of polaritonpolariton interactions on the excitation spectrum of a nonequilibrium condensate in a periodic potential, Phys. Rev. B 87, 155423 (2013).

[51] N. Peyghambarian, H. M. Gibbs, J. L. Jewell, A. Antonetti, A. Migus, D. Hulin, and A. Mysyrowicz, Blue Shift of the Exciton Resonance due to Exciton-Exciton Interactions in a MultipleQuantum-Well Structure, Phys. Rev. Lett. 53, 2433 (1984). 